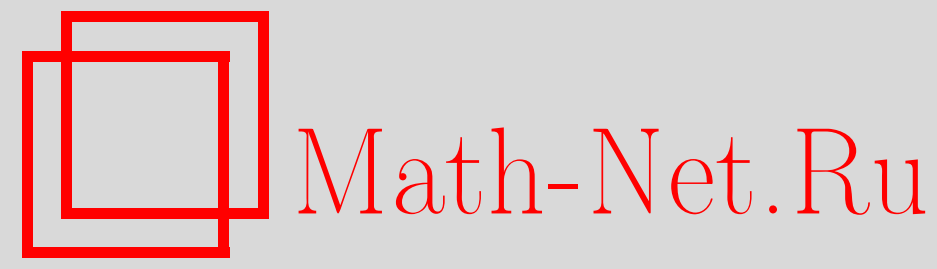

Т. К. Фахрутдинова, Об условиях вырождения ветвящихся процессов в случайной среде с зависящим от состояния размножением, Теория вероятн. и ее примен., 2008, том 53, выпуск 4, 818-822

DOI: https://doi.org/10.4213/tvp2469

Использование Общероссийского математического портала MathNet.Ru подразумевает, что вы прочитали и согласны с пользовательским соглашением

http://www . mathnet.ru/rus/agreement

Параметры загрузки:

IP: 35.174 .16 .151

26 апреля 2023 г., 16:41:43

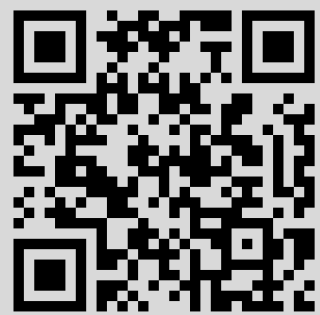


(c) $2008 \mathrm{r}$.

ФАХРУТДИНОВА Т. К.*

\section{ОБ УСЛОВИЯХ ВЫРОЖДЕНИЯ ВЕТВЯЩИХСЯ ПРОЩЕССОВ В СЛУЧАЙНОЙ СРЕДЕ С ЗАВИСЯІЩИМ ОТ СОСТОЯНИЯ РАЗМНОЖЕНИЕМ}

В работе изучаются условия вырождения, зависящего от состояния ветвящегося процесса в случайной среде. Исследование основано на применении критерия Керстинга для моделей роста к логарифму ветвящегося процесса.

Ключевые слова и фразы: ветвяшийся процесс, зависимость от состояния, случайная среда, моментное неравенство для сумм.

1. Введение. Рассмотрим семейство вероятностных производяших функций $f(s ; y, m)$, зависящее от действительного параметра $y$ и целого неотрицательного $m$, и «случайную среду» $\bar{\eta}=\left(\eta_{1}, \eta_{2}, \ldots\right)$ - последовательность независимых одинаково распределенных случайных величин (н.о.р. с.в.) с распределением вероятностей $\mu$, заданньгх на некотором измеримом пространстве $(\mathscr{Y}, \mathscr{B})$. Ветвящийся процесс в случайной среде с размножением, зависяшим от состояния, зададим рекуррентно:

$$
Z_{0}=1, \quad Z_{n+1}=\sum_{i=1}^{Z_{n}} \zeta_{i n}\left(Z_{n}\right)
$$

(где сумма по пустому множеству индексов полагается нулевой), предполагая, что при условии $Z_{k}, \eta_{k}, k=1, \ldots, n$, случайные величины $\zeta_{\text {in }}\left(Z_{n}\right), i=1, \ldots, Z_{n}$, независимы и одинаково распределены с производяшими функциями $f\left(s ; \eta_{n}, Z_{n}\right)$, а для различных $n$ последовательности $\left\{\zeta_{\text {in }}(m), i=1, \ldots, m\right\}$ условно независимы. Иначе говоря, $Z_{n}$ есть марковская цепь с вероятностями перехода $p_{i j}:=\mathbf{E} p_{j}(\eta, i), i, j \in \mathbf{Z}^{+}$, где $p_{j}(y, i)$ есть $j$-й коэффициент в разложении функции $(f(s ; y, i))^{i}$ в степенной ряд, $\eta$ - общее обозначение для с.в. $\eta_{n}$. В настоящей работе находятся условия, при которых происходит п.н. поглощение процесса $Z_{n}$ в состоянии 0 или, напротив, процесс с положительной вероятностью уходит на бесконечность. Для этого к процессу $X_{n}:=\ln Z_{n}$ применяется критерий возвратности и невозвратности Керстинга [1], основанный на асимптотике функции

$$
h(x):=2 x \frac{\mathbf{E}\left(\Delta X_{n} \mid X_{n}=x\right)}{\mathbf{D}\left(\Delta X_{n} \mid X_{n}=x\right)}, \quad \text { где } \Delta X_{n}=X_{n+1}-X_{n} .
$$

Если $\lim \sup _{x \rightarrow \infty} h(x)<1$, то при некоторьх дополнительных ограничениях процесс $X_{n}$ п.н. возврашается в заданную ограниченную окрестность нуля, а если $\liminf _{x \rightarrow \infty} h(x)>1$, то он с положительной вероятностью стремится к бесконечности. Вывод асимптотики условных моментов прирашений $\Delta X_{n}$, входящих в (1), основан на следующих соображениях. В предположении $0<f^{\prime}(1 ; \eta, m)<\infty$ п.н., $m=1,2, \ldots$, имеем: $\mathbf{E}\left(Z_{n+1} \mid \eta_{n}, Z_{n}\right)=Z_{n} f^{\prime}\left(1 ; \eta_{n}, Z_{n}\right)$, а при условии $Z_{n}=m, \eta_{n}$

$$
\frac{Z_{n+1}}{m} \longrightarrow f^{\prime}\left(1 ; \eta_{n}, m\right) \equiv \mathbf{E}\left(\zeta_{\text {in }}(m) \mid \eta_{n}\right) \quad \text { п.н. при } m \rightarrow \infty \text {. }
$$

Можно ожидать, что поведение переходных вероятностей марковской цепи $X_{n}$ в окрестности бесконечно удаленной точки фазового пространства будет близко к таковому для марковской цепи $Y_{n}$, заданной рекуррентным соотношением

$$
Y_{n+1}=Y_{n}+\ln f^{\prime}\left(1 ; \eta_{n}, e^{Y_{n}}\right)
$$

* Московский государственный университет им. М.В. Ломоносова, механикоматематический факультет, Ленинские горы 1, 119992 Москва, Россия; е-mail: fakhrutdinova@mail.ru 
а это поведение (с точностью до граничных условий в нуле) является определяющим для возвратности или невозвратности процесса. В работе [2] показано, что имеет место следуюшее соотношение:

$$
h(x) \sim \widetilde{h}(x):=2 x \frac{\mathbf{E}\left(\Delta Y_{n} \mid Y_{n}=x\right)}{\mathbf{D}\left(\Delta Y_{n} \mid Y_{n}=x\right)} \quad \text { при } x \rightarrow \infty
$$

здесь и далее символ $\sim$ обозначает, что отношение соединенньх им величин стремится к 1. Тем самым, критерий возвратности и невозвратности выражен через исходные данные модели. Наиболее ограничительным предположением, при котором в [2] выведена эквивалентность (4), является конечность моментов $\mathbf{E}\left|\zeta_{\text {in }}(m)\right|^{2+\delta}$, $\delta>0$. В настоящей работе это условие заменяется требованием конечности моментов $\mathbf{E} \varphi\left(\zeta_{\text {in }}(m)\right)$, где

$$
\varphi(x)=|x| \ln ^{3+\delta}\left(|x|+e^{2+\delta}\right), \quad \delta>0 .
$$

Это предположение, как видно, близко к требованию $\mathbf{E}\left|\zeta_{\text {in }}(m)\right|<\infty$, которое, ввиду соотношения (2), лежит в основе применяемого подхода. В наших рассуждениях существенную роль выполняет следующее обобщение неравенства Бара-Эссеена.

Теорема 1 [3]. Пусть функиия $H(x), x \in(-\infty,+\infty)$, слабо выпукла, т.e. удовлетворяет следуюиим условиям:

1) $H(x)$ - выпуклая четная функиия;

2) $H(0)=0$ и $H(x)$ не убывает на $(0,+\infty)$;

3) $H(x)$ дифФеренцируема всюду, кроме, быть может, точки $x=0$, в которой предел $\lim _{x \rightarrow+0} H^{\prime}(x)=H^{\prime}(+0)$ существует ( $u$, в силу выпуклости, конечен);

4) $H^{\prime}(x)$ - вогнутая функиия на $(0,+\infty)$.

Пусть последовательность $W_{0}=0, W_{1}, \ldots, W_{n}$ является мартингалом $u X_{k}=$ $W_{k}-W_{k-1}, k=1, \ldots, n$, обозначают соответствуюиие мартингал-разности. Если $\mathbf{E} H\left(X_{k}\right)<\infty, k=1, \ldots, n, m o$

$$
\mathbf{E} H\left(W_{n}\right) \leqslant 4 \sum_{k=1}^{n} \mathbf{E} H\left(X_{k}\right)
$$

2. Предположения и результаты. Положим

$$
\begin{aligned}
X_{n} & :=L\left(Z_{n}\right), \quad \text { где } L(z):= \begin{cases}\ln z, & z>e, \\
e^{-1} z, & z \leqslant e,\end{cases} \\
\alpha_{m}(\eta) & :=f^{\prime}(1 ; \eta, m)=\mathbf{E} \zeta_{\text {in }}(m), \quad \lambda_{m}(\eta):=\ln \alpha_{m}(\eta), \quad p=2+\delta, \quad \delta>0, \\
\rho_{m}(\eta) & :=\mathbf{E}\left(\left|\zeta_{i n}(m)-\alpha_{m}(\eta)\right| \ln ^{p+1}\left(\left|\zeta_{\text {in }}(m)-\alpha_{m}(\eta)\right|+e^{p}\right)\right) \alpha_{m}(\eta)^{-1} .
\end{aligned}
$$

Как отмечено в работе [2], малые значения $\alpha_{m}(\eta)$ могут привести к вырождению процесса вне зависимости от других его характеристик. Поэтому далее рассматриваются моменты с.в., суженные на событие $\mathscr{A}_{m}(\eta)=\left\{\alpha_{m}(\eta) m>2 e\right\}$. Введем также события $\overline{\mathscr{A}}_{m}(\eta)=\left\{\alpha_{m}(\eta) m \leqslant 2 e\right\}$.

Положим $\mathbf{E}_{m} X:=\mathbf{E}\left(X \mid Z_{n}=m\right), \widehat{X}:=X \chi\left(\mathscr{A}_{m}(\eta)\right)$, где $\chi(A)$ есть индикатор события $A$, а в качестве $X$ будут выступать различные связанные с процессом $X_{n}$ величины. Далее предполагается, что для любого $m$

$$
0<\mathbf{D} \widehat{\lambda}_{m}(\eta)<\infty, \quad \mathbf{E} \frac{\widehat{\rho}_{m}(\eta)}{\ln \left(\alpha_{m}(\eta) m\right)}<\infty .
$$

Следующие предположения соответствуют условиям Керстинга, если заменить прирашение $\Delta X_{n}$ на его аппроксимацию $\ln f^{\prime}\left(1 ; \eta_{n}, e^{X_{n}}\right)$ (см. (3)):

$$
\mathbf{E}\left|\widehat{\lambda}_{m}(\eta)-\mathbf{E} \widehat{\lambda}_{m}(\eta)\right|^{p}=O(1) \mathbf{D}\left(\widehat{\lambda}_{m}(\eta)\right)^{p / 2}
$$




$$
\begin{gathered}
\mathbf{E} \widehat{\lambda}_{m}(\eta)=o(1) \ln m, \\
\mathbf{E} \widehat{\lambda}_{m}(\eta)=O(1) \ln m(\ln \ln m)^{-\beta}, \quad \beta>\frac{2}{\delta} .
\end{gathered}
$$

Следуюшие условия обеспечивают необходимую точность приближения моментов прирашений $\Delta X_{n}$ при условии $Z_{n}=m$ моментами с.в. $\widehat{\lambda}_{m}(\eta)$ :

$$
\begin{gathered}
\mathbf{E} \frac{\hat{\rho}_{m}(\eta)}{\ln ^{p-1}\left(\alpha_{m}(\eta) m\right)}=o(1) \frac{\mathbf{D} \hat{\lambda}_{m}(\eta)}{\ln m}, \\
\ln m \cdot \mathbf{P}\left(\overline{\mathscr{A}}_{m}(\eta)\right)=o(1) \frac{\mathbf{D} \hat{\lambda}_{m}(\eta)}{\ln m}, \\
\ln ^{p} m \cdot \mathbf{P}\left(\overline{\mathscr{A}}_{m}(\eta)\right)+\mathbf{E} \frac{\widehat{\rho}_{m}(\eta)}{\ln \left(\alpha_{m}(\eta) m\right)}=O(1)\left(\mathbf{D} \hat{\lambda}_{m}(\eta)\right)^{p / 2}
\end{gathered}
$$

Следуюшие условия также призваны обеспечить применимость критерия Керстинга (cp. (B2a), (B2b)):

$$
\begin{gathered}
\mathbf{E} \frac{\widehat{\rho}_{m}(\eta)}{\ln ^{p}\left(\alpha_{m}(\eta) m\right)}+\ln m \cdot \mathbf{P}\left(\overline{\mathscr{A}}_{m}(\eta)\right)=o(1) \ln m, \\
\mathbf{E} \frac{\widehat{\rho}_{m}(\eta)}{\ln ^{p}\left(\alpha_{m}(\eta) m\right)}+\ln m \cdot \mathbf{P}\left(\overline{\mathscr{A}}_{m}(\eta)\right)=O(1) \frac{\ln m}{(\ln \ln m)^{\beta}} .
\end{gathered}
$$

Теорема 2. B предположениях (A), (B1), (B2a), (C), (Da) имеют место coотношения:

$$
\begin{gathered}
\mathbf{E}_{m} \Delta X_{n}=\mathbf{E} \widehat{\lambda}_{m}(\eta)+o(1) \frac{\mathbf{D} \widehat{\lambda}_{m}(\eta)}{\ln m}, \\
\mathbf{E}_{m} \Delta X_{n}=o(1) \ln m, \quad \mathbf{D}_{m}\left(\Delta X_{n}\right) \sim \mathbf{D} \widehat{\lambda}_{m}(\eta), \\
\mathbf{E}_{m}\left|\Delta X_{n}-\mathbf{E}_{m} \Delta X_{n}\right|^{p}=O(1)\left(\mathbf{D} \widehat{\lambda}_{m}(\eta)\right)^{p / 2}, \\
h(\ln m) \equiv \frac{2 \ln m \cdot \mathbf{E}_{m}\left(\Delta X_{n}\right)}{\mathbf{D}_{m}\left(\Delta X_{n}\right)}=\frac{2 \ln m \cdot \mathbf{E} \widehat{\lambda}_{m}(\eta)}{\mathbf{D} \widehat{\lambda}_{m}(\eta)}+o(1) .
\end{gathered}
$$

Eсли $\lim \sup _{m \rightarrow \infty} h(\ln m)<1$, то выполнены все условия теоремы 1 работы [1] $u$, следовательно, $\mathbf{P}\left\{X_{n} \rightarrow 0\right\}=1$. Eсли вместо (B2a), (Da) выполнены более жесткие предположения (B2b), (Db) $u \liminf _{m \rightarrow \infty} h(\ln m)>1$, mо $\mathbf{E}_{m} \Delta X_{n}=$ $O(1) \ln m \cdot(\ln \ln m)^{-\beta}$, выполнены все условия теоремы 2 работы [1] $u$, следовательно, $\mathbf{P}\left\{X_{n} \rightarrow \infty\right\}>0, \mathbf{P}\left\{X_{n} \rightarrow \infty\right.$ или $\left.X_{n} \rightarrow 0\right\}=1$.

\section{3. Оиенки логарифмических моментов сумм случайных величин.}

3.1. Суммы независимых одинаково распределенных случайных величин. Пусть $\left\{\zeta_{1}, \zeta_{2}, \ldots\right\}$ - последовательность неотрицательных н.о.р. с.в. с конечным математическим ожиданием $\alpha:=\mathbf{E} \zeta_{1}$. Положим $S_{m}=\sum_{k=1}^{m} \zeta_{k}, F_{m}(x)=\mathbf{P}\left\{S_{m} \leqslant x\right\}$, $F_{m}^{0}(y)=\mathbf{P}\left\{S_{m}-\alpha m \leqslant y\right\}$.

Лемма 1. При $\alpha m \geqslant 2 e, m \geqslant 2$ имеют место неравенства:

$$
\begin{gathered}
\left|\int(L(x)-\ln (\alpha m)) d F_{m}(x)\right| \leqslant C_{1} \alpha^{-1} \mathbf{E} \varphi\left(\zeta_{1}-\alpha\right)(\ln (\alpha m))^{-p}, \\
\int(L(x)-\ln (\alpha m))^{2} d F_{m}(x) \leqslant C_{2} \alpha^{-1} \mathbf{E} \varphi\left(\zeta_{1}-\alpha\right)(\ln (\alpha m))^{-(p-1)}, \\
\int|L(x)-\ln (\alpha m)|^{p} d F_{m}(x) \leqslant C_{3} \alpha^{-1} \mathbf{E} \varphi\left(\zeta_{1}-\alpha\right)(\ln (\alpha m))^{-1},
\end{gathered}
$$

где $C_{1}, C_{2}, C_{3}$ - некоторые константы, зависящие от $p$. 
Докажем для примера неравенство (9).

1) Перейдем от переменной $x$ к $y=x-\alpha m$ и рассмотрим интервал интегрирования $-\alpha m \leqslant y \leqslant-\alpha m / 2$. На этом интервале $\varphi(y)$ неотрицательна и имеет минимум при $y=-\alpha m / 2$, а также имеет место неравенство $|L(y+\alpha m)-\ln (\alpha m)| \leqslant \ln (\alpha m)$. Поэтому можно оценить интеграл (9) сверху следуюшим образом:

$$
\begin{aligned}
\int_{-\alpha m}^{-\alpha m / 2}(L(y+\alpha m)-\ln (\alpha m))^{2} d F_{m}^{0}(y) & \leqslant \frac{\ln ^{2}(\alpha m)}{\varphi(-\alpha m / 2)} \int_{-\alpha m}^{-\alpha m / 2} \varphi(y) d F_{m}^{0}(y) \\
& \leqslant \frac{\ln ^{2}(\alpha m)}{\varphi(-\alpha m / 2)} \mathbf{E} \varphi\left(S_{m}-\alpha m\right) .
\end{aligned}
$$

Легко проверить, что функция $\varphi(y)$ слабо выпукла (см. теорему 1 ). Применяя неравенство (6) к случайному блужданию $W_{m}=S_{m}-\alpha m$, получаем: $\mathbf{E} \varphi\left(S_{m}-\alpha m\right) \leqslant$ $4 m \mathbf{E} \varphi\left(\zeta_{1}-\alpha\right)$.

Так как $\ln (\alpha m) \leqslant 2 \ln (\alpha m / 2)$ при $\alpha m \geqslant 2 e$, то имеет место оценка:

$$
\frac{4 m \mathbf{E} \varphi\left(\zeta_{1}-\alpha\right) \ln ^{2}(\alpha m)}{(\alpha m / 2) \ln ^{p+1}\left(\alpha m / 2+e^{p}\right)} \leqslant 2^{p+4} \alpha^{-1} \mathbf{E} \varphi\left(\zeta_{1}-\alpha\right)(\ln (\alpha m))^{-(p-1)}
$$

2) Рассмотрим интеграл в (9) по промежутку $-\alpha m / 2 \leqslant y \leqslant \alpha m / 2$. При $y \geqslant$ $-\alpha m / 2$ имеем $L(y+\alpha m)=\ln (y+\alpha m)$, так что задача сводится к оценке интеграла

$$
\int_{-\alpha m / 2}^{\alpha m / 2}(\ln (y+\alpha m)-\ln (\alpha m))^{2} d F_{m}^{0}(y)=\int_{-\alpha m / 2}^{\alpha m / 2} \ln ^{2}\left(1+\frac{y}{\alpha m}\right) d F_{m}^{0}(y) .
$$

Нетрудно показать, что при $|y| \leqslant \alpha m / 2, \alpha m \geqslant 2 e$, выполняется неравенство $|\ln (1+y /(\alpha m))| \leqslant C(\ln (\alpha m))^{-p} \ln ^{p+1}\left(|y|+e^{p}\right)$, где $C$ - некоторая константа, не зависящая от $m$. Так как при $|y| \leqslant \alpha m / 2$ выполнено неравенство $\ln ^{2}(1+y /(\alpha m)) \leqslant$ $2|y|(\alpha m)^{-1}|\ln (1+y /(\alpha m))|$, то

$$
\begin{gathered}
\int_{-\alpha m / 2}^{\alpha m / 2} \ln ^{2}\left(1+\frac{y}{\alpha m}\right) d F_{m}^{0}(y) \leqslant \frac{2 C}{\alpha m} \int_{-\alpha m / 2}^{\alpha m / 2} \frac{\varphi(y)}{\ln ^{p}(\alpha m)} d F_{m}^{0}(y) \\
\leqslant \frac{2 C \mathbf{E} \varphi\left(S_{m}-\alpha m\right)}{\alpha m \ln ^{p}(\alpha m)} \leqslant 8 C \alpha^{-1} \mathbf{E} \varphi\left(\zeta_{1}-\alpha\right)(\ln (\alpha m))^{-p}
\end{gathered}
$$

3) Рассмотрим интеграл по промежутку $y \geqslant \alpha m / 2$. Воспользуемся оценкой $\ln (1+$ $y /(\alpha m)) \leqslant y /(\alpha m)$ и проведем интегрирование по частям:

$$
\begin{gathered}
\int_{\alpha m / 2}^{\infty} \ln ^{2}\left(1+\frac{y}{\alpha m}\right) d F_{m}^{0}(y) \leqslant \frac{1}{\alpha m} \int_{\alpha m / 2}^{\infty} y \ln \left(1+\frac{y}{\alpha m}\right) d F_{m}^{0}(y) \leqslant \frac{1}{2}\left(1-F_{m}^{0}\left(\frac{\alpha m}{2}\right)\right) \\
+\frac{1}{\alpha m} \int_{\alpha m / 2}^{\infty}\left(1-F_{m}^{0}(y)\right)\left[\ln \left(1+\frac{y}{\alpha m}\right)+\frac{y}{\alpha m+y}\right] d y .
\end{gathered}
$$

Для первого слагаемого воспользуемся оценкой

$$
\frac{1}{2}\left(1-F_{m}^{0}\left(\frac{\alpha m}{2}\right)\right) \leqslant \frac{\mathbf{E} \varphi\left(S_{m}-\alpha m\right)}{2 \varphi(\alpha m / 2)} \leqslant 2^{p+2} \alpha^{-1} \mathbf{E} \varphi\left(\zeta_{1}-\alpha\right)(\ln (\alpha m))^{-(p+1)} .
$$

Второе слагаемое не превосходит

$$
\begin{aligned}
& \frac{4 m \mathbf{E} \varphi\left(\zeta_{1}-\alpha\right)}{\alpha m} \int_{\alpha m / 2}^{\infty} \frac{1}{\varphi(y)}\left[\ln \left(1+\frac{y}{\alpha m}\right)+\frac{y}{\alpha m+y}\right] d y \\
& \leqslant \frac{4 \mathbf{E} \varphi\left(\zeta_{1}-\alpha\right)}{\alpha} \int_{\alpha m / 2}^{\infty} \frac{2 \ln \left(y+e^{p}\right)}{y \ln ^{p+1}\left(y+e^{p}\right)} d y \leqslant \frac{8 \mathbf{E} \varphi\left(\zeta_{1}-\alpha\right)}{\alpha} \int_{\alpha m / 2}^{\infty} \frac{d(\ln y)}{\ln ^{p} y} \\
& \leqslant 2^{p+2} \alpha^{-1} \mathbf{E} \varphi\left(\zeta_{1}-\alpha\right)(\ln (\alpha m))^{-(p-1)} .
\end{aligned}
$$

Собирая все оценки, получаем неравенство (9). 
3.2. Схема доказательства теоремы 2.

Лемма 2. При условиях (C) $и m \geqslant 3$ справедливы неравенства:

$$
\begin{aligned}
\left|\mathbf{E}_{m} \Delta X_{n}-\mathbf{E} \hat{\lambda}_{m}(\eta)\right| \leqslant & B_{1}\left\{\mathbf{E} \frac{\hat{\rho}_{m}(\eta)}{\ln ^{p}\left(\alpha_{m}(\eta) m\right)}+\ln m \cdot \mathbf{P}\left(\overline{\mathscr{A}}_{m}(\eta)\right)\right\} \\
\left|\mathbf{D}_{m} \Delta X_{n}-\mathbf{D} \hat{\lambda}_{m}(\eta)\right| \leqslant & B_{2} \max \left\{\ln m,\left|\mathbf{E} \hat{\lambda}_{m}(\eta)\right|\right\} \\
& \times\left\{\mathbf{E} \frac{\hat{\rho}_{m}(\eta)}{\ln ^{p-1}\left(\alpha_{m}(\eta) m\right)}+\ln m \cdot \mathbf{P}\left(\overline{\mathscr{A}}_{m}(\eta)\right)\right\} \\
\mathbf{E}_{m}\left|\Delta X_{n}-\mathbf{E}_{m} \Delta X_{n}\right|^{p} \leqslant & B_{3}\left\{\mathbf{E} \frac{\hat{\rho}_{m}(\eta)}{\ln \left(\alpha_{m}(\eta) m\right)}+\mathbf{E}\left|\widehat{\lambda}_{m}(\eta)-\mathbf{E} \widehat{\lambda}_{m}(\eta)\right|^{p}\right. \\
& \left.+\ln ^{p} m \cdot \mathbf{P}\left(\overline{\mathscr{A}}_{m}(\eta)\right)\right\}
\end{aligned}
$$

где $B_{1}, B_{2}, B_{3}$ - некоторые константы.

Оценки (11)-(13) можно получить, заменяя в неравенствах (8)-(10) $F_{m}(x)$ на $F_{m}(x ; y)$ и $\alpha$ на $\alpha_{m}(y)$, интегрируя полученные выражения относительно меры $\mu(d y)$ по области $\left\{y: \alpha_{m}(y) m>2 e\right\}$ и применяя следующее утверждение.

Лемма 3 ([2, следствие 4.1]). Для $m \geqslant 3 u 1 \leqslant r \leqslant p$

$$
\mathbf{E}_{m}\left(\left|\Delta X_{n}\right|^{r} ; \overline{\mathscr{A}}_{m}(\eta)\right) \leqslant 2^{p}\left(c_{p}+\ln ^{r} m\right) \mathbf{P}\left(\overline{\mathscr{A}}_{m}(\eta)\right)
$$

с некоторой абсолютной постоянной $c_{p}>1$.

Комбинируя неравенство (11) с условиями (C1), (C2), (B2a), (Da), получаем: $\mathbf{E}_{m} \Delta X_{n}=\mathbf{E} \widehat{\lambda}_{m}(\eta)+o(1)(\ln m)^{-1} \mathbf{D} \widehat{\lambda}_{m}(\eta), \mathbf{E}_{m} \Delta X_{n}=o(1) \ln m$. Если вместо (B2a), (Da) выполнены ограничения (B2b), (Db), то из (11) следует, что $\mathbf{E}_{m} \Delta X_{n}=$ $O(1) \ln m \cdot(\ln \ln m)^{-\beta}$. Из неравенства (12) и предположений (B2a), (C1), (C2) получаем: $\mathbf{D}_{m}\left(\Delta X_{n}\right) \sim \mathbf{D} \widehat{\lambda}_{m}(\eta)$. Наконец, из неравенства (13) и предположений (B1), (C3) следует соотношение $\mathbf{E}_{m}\left|\Delta X_{n}-\mathbf{E}_{m} \Delta X_{n}\right|^{p}=O(1)\left(\mathbf{D} \hat{\lambda}_{m}(\eta)\right)^{p / 2}$.

Таким образом, выполнены все условия, необходимые для применения критерия Керстинга [1], тем самым получено представление (7) для функции $h(\ln m)$ и теорема 2 доказана.

Автор выражает М. В. Козлову благодарность за руководство. Автор благодарен рецензенту, обратившему внимание на работу [3].

\section{СПИСОК ЛИТЕРАТУРЫ}

1. Kersting G. On recurrence and transience of growth models. - J. Appl. Probab., 1986 , v. 23 , p. $614-625$.

2. Альбеверио $C$., Козлов $M . B$. О рекуррентности и транзиентности зависящих от состояния ветвяшихся процессов в случайной среде. - Теория вероятн. и ее примен., 2003, т. 48 , в. 4 , с. $641-660$.

3. Ватутин B.A., Топчий B.A. Максимум критических процессов ГальтонаВатсона и непрерывные слева случайные блуждания. - Теория вероятн. и ее примен., 1997, т. 42 , в. 1, с. 21-34.

Поступила в редакцию 1.II.2008

Исправленный вариант 26.VI. 2008 